\title{
THE FUNDAMENTAL SOLUTION OF A DEGENERATE PARTIAL DIFFERENTIAL EQUATION OF PARABOLIC TYPE $\left({ }^{1}\right)$
}

BY

\section{MARIA WEBER}

1. Introduction. The equations studied in this paper arise in the probability treatment of diffusion problems and were first introduced by Kolmogoroff [1] $\left({ }^{2}\right)$. Kolmogoroff showed that under certain conditions the probability density of a system with $2 n$ degrees of freedom satisfies a parabolic differential equation of Fokker-Planck type. The ordinary FokkerPlanck equation in $2 n$ dimensions is

$$
\sum_{i, j}^{n} a_{i j} \frac{\partial^{2} u}{\partial x_{i} \partial x_{j}}+\sum_{i}^{n}\left(x_{i} \frac{\partial u}{\partial y_{i}}+a_{i} \frac{\partial u}{\partial x_{i}}\right)+a u+\frac{\partial u}{\partial t}=0 \quad\left(a_{i j}=a_{j i}\right)
$$

$a_{i j}, a_{i}, a$ are functions of $x, y, t$. It is degenerate in the sense that the second derivatives in $y$ do not appear in the equation. The $2 n$-dimensional space is the phase space of a system, where $y$ is the position and $x$ the velocity vector. For a more recent discussion of stochastic processes giving rise to equations of that type, see S. Chandrasekhar [2]. The more general equation

$$
\sum_{i, j}^{n} a_{i j} \frac{\partial^{2} u}{\partial \xi_{i} \partial \xi_{i}}+\sum_{i}^{n}\left(b_{i} \frac{\partial u}{\partial \eta_{i}}+a_{i} \frac{\partial u}{\partial \xi_{i}}\right)+a u+\frac{\partial u}{\partial \tau}=0
$$

can be reduced to (1.1) by the substitution $x_{k}=b_{k}(\xi, \eta, \tau)$ provided

$$
\frac{\partial b_{i}}{\partial \xi_{k}}, \frac{\partial b_{i}}{\partial \eta_{k}}, \frac{\partial b_{i}}{\partial \tau}
$$

exist for all $i$ and $k$ and the transformation

$$
x_{k}=b_{k}(\xi, \eta, \tau), \quad y_{k}=\eta_{k}, \quad t=\tau
$$

represents a continuous one-to-one mapping of the $\xi, \eta, \tau$-plane on the $x, y, t$ plane. Here the relation between the position $\eta$ and the velocity $\xi$ is given by $\dot{\eta}_{i}=b_{i}$.

The construction of a solution of (1.1) depends on the determination of the fundamental solution. It is the purpose of this paper to obtain the fundamental solution of (1.1) for any given open region $R$ of phase space under

Received by the editors August 14, 1950.

(1) A Ph.D. dissertation submitted to Cornell University.-The research was done in connection with an ONR project in development of probability theory at Cornell University.The author thanks Professor W. Feller for his valuable help in the preparation of this paper.

(2) Numbers in brackets refer to the bibliography at the end of the paper. 
certain conditions on the coefficients; these conditions will be given in $\S 3$. (The region $R$ is not necessarily bounded, it may cover the whole phasespace.) Our development follows closely the methods of Feller [3] and Dressel $[4 ; 5]$, going in some essentials back to Gevrey $[6 ; 7]$.

Notations. Throughout the text the following notations are used: When there is no misunderstanding multiple integrals will be indicated by a single integral sign. We shall write $d x$ and $d y$ for $d x_{1} \cdots d x_{n}$ and $d y_{1} \cdots d y_{n}$ respectively. Integration with respect to $t$ will always be indicated separately. The notation $x$ will be used for $x_{1} \cdots x_{n}$ and similarily $y, \xi, \eta, \mu, \nu$ represent points in $n$-space. All summations, unless otherwise indicated, extend from 1 to $n$.

2. Definition of the fundamental solution and the problem of uniqueness. Let $(1.1)$ be defined over some open region $R$ in phase-space $(x, y)$ and for $t_{0} \leqq t \leqq t_{1}$, with uniformly bounded and continuous coefficients $a_{i j}, a_{i}, a$. Let $\partial a_{i j} / \partial x_{i}$ be uniformly bounded and continuous. We define the fundamental solution $u$ of (1.1) by the following three properties:

(I) For $t_{0} \leqq t<\tau \leqq t_{1}$ and each pair of points $(x, y)$ and $(\xi, \eta)$ in $R, u(x, y, t$; $\xi, \eta, \tau)$ is a regular solution of (1.1), that is, it possesses, as a function of $(x, y, t)$, the continuous derivatives occurring in equation (1.1).

(II) For $x=\xi, y=\eta, t=\tau$ the function $u(x, y, t ; \xi, \eta, \tau)$ possesses a singularity such that for every subregion $D$ of $R$ and every continuous bounded function $f(x, y)$

(2.1) $\lim _{t \rightarrow \tau-} \int_{D} u(x, y, t ; \xi, \eta, \tau) f(x, y) d x d y= \begin{cases}f(\xi, \eta) & \text { if }(\xi, \eta) \text { is interior to } D, \\ 0 & \text { if }(\xi, \eta) \text { is exterior to } D .\end{cases}$

(III) For fixed $\xi, \eta, \tau, t$ with $t_{0} \leqq t<\tau \leqq t_{1}$ the functions $u(x, y, t ; \xi, \eta, \tau)$ and $x_{i} u(x, y, t ; \xi, \eta, \tau)$ are absolutely integrable over $R$ and $\partial u / \partial x_{i}$ are bounded.

The equation

$$
L^{*}(u)=\sum_{i, i} \frac{\partial^{2} a_{i j} u}{\partial x_{i} \partial x_{j}}-\sum_{i}\left(\frac{\partial a_{i} u}{\partial x_{i}}+x_{i} \frac{\partial u}{\partial y_{i}}\right)+a u-\frac{\partial u}{\partial \tau}=0
$$

defines the adjoint to (1.1). In $\$ 3$ we shall give sufficient conditions on the coefficients of (1.1) to ensure the existence of a fundamental solution. These conditions will automatically entail the existence of a fundamental solution of (2.2).

We shall now give a uniqueness theorem for the fundamental solution, provided $R$ is the entire phase space

$$
S\left\{\begin{array}{l}
-\infty<x_{i}<+\infty, \\
-\infty<y_{i}<+\infty,
\end{array} \quad i=1,2, \cdots, n .\right.
$$

THEOREM 1. Under the assumption of existence of a fundamental solution of 
(2.2), $u(x, y, t ; \xi, \eta, \tau)$, as defined by conditions I $\rightarrow$ III with $R \equiv S$, satisfies equation (2.2) in the variables $\xi, \eta$, and $\tau$ and as a consequence is uniquely determined.

Proof. The proof is omitted because it follows the same lines as for the ordinary parabolic equation (cf. Dressel [5]).

Corollary.

$$
\int_{S} u(x, y, t ; \xi, \eta, \tau) u(\mu, \nu, \lambda ; x, y, t) d x d y=u(\mu, \nu, \lambda ; \xi, \eta, \tau) .
$$

3. The fundamental solution of equation (1.1). We determine the fundamental solution as the solution of an integral equation. We assume that in $R$ and for $t_{0} \leqq t \leqq t_{1}$ the coefficients of (1.1) satisfy the following conditions:

(a) The functions $\partial a_{i j} / \partial t, \partial^{2} a_{i j} / \partial x_{k} \partial x_{e}, \partial a_{i} / \partial x_{k}, a_{i}, a, \partial a_{i j} / \partial y_{k}$ satisfy a local Lipschitz condition of order $\gamma, 0<\gamma$, and are uniformly bounded.

(b) The characteristic roots of the symmetric matrix $\left\|a_{i j}\right\|$ are positive and uniformly bounded both above and away from zero.

Let $A_{i k}$ denote the cofactor of $a_{i k}$ divided by the determinant $A$. Because of condition (b), $A$ is bounded above and below and so are the characteristic roots of $\left\|A_{i k}\right\|$. Then as an immediate consequence of (b) we have:

Lemma 1. There exist positive constants $d_{1}$ and $d_{2}$ such that for all $u_{i}$ and all $(x, y, t)$ in $R$

$$
\begin{aligned}
& d_{1} \sum_{i} u_{i}^{2} \leqq \sum_{i, k} a_{i k} u_{i} u_{k} \leqq d_{2} \sum_{i} u_{i}^{2}, \\
& d_{1} \sum_{i} u_{i}^{2} \leqq \sum_{i, k} A_{i k} u_{i} u_{k} \leqq d_{2} \sum_{i} u_{i}^{2} .
\end{aligned}
$$

$d_{1}$ is the greatest lower bound of the characteristic roots of both $\left\|a_{i k}\right\|$ and $\left\|A_{i k}\right\|$ and $d_{2}$ the least upper bound.

In the case of equation (1.2) additional assumptions are to be made on derivatives of the $b_{i}$ 's up to the third order and $a_{i j}$ is to be replaced by

$$
\bar{a}_{i j}=\frac{1}{2} \sum_{k, e} a_{k \bullet}\left[\frac{\partial b_{e}}{\partial x_{i}} \frac{\partial b_{k}}{\partial x_{j}}+\frac{\partial b_{e}}{\partial x_{j}} \frac{\partial b_{k}}{\partial x_{i}}\right] .
$$

We now proceed to prove the following theorem.

THEOREM 2. Under assumptions (a) and (b) there exists a fundamental solution of (1.1). In case $R$ is the entire phase space this fundamental solution is unique and satisfies equation (2.2).

We need some preliminary results:

The equation 


$$
L(u)=\frac{\partial^{2} u}{\partial x^{2}}+x \frac{\partial u}{\partial y}+\frac{\partial u}{\partial t}=0
$$

has the fundamental solution

$$
\begin{aligned}
F(x, y, t ; \xi, \eta, \tau)=3^{1 / 2} 2^{-1} \pi^{-1}(\tau-t)^{-2} & \exp \left[-\frac{(\xi-x)^{2}}{4(\tau-t)}\right. \\
-3 & \left.\frac{\left\{\eta-y-2^{-1}(\tau-t)(\xi+x)\right\}^{2}}{(\tau-t)^{3}}\right]
\end{aligned}
$$

given by Kolmogoroff [1], which satisfies all the conditions in $\S 2$. We use this function in the construction of the first approximation of the fundamental solution of (1.1).

Let

$$
\begin{aligned}
& R_{i k}(x, y, t ; \xi, \eta, \tau)=\frac{\left(\xi_{i}-x_{i}\right)\left(\xi_{k}-x_{k}\right)}{4(\tau-t)} \\
& \quad+3 \frac{\left\{\eta_{i}-y_{i}-2^{-1}(\tau-t)\left(\xi_{i}+x_{i}\right)\right\}\left\{\eta_{k}-y_{k}-2^{-1}(\tau-t)\left(x_{k}+\xi_{k}\right)\right\}}{(\tau-t)^{3}} .
\end{aligned}
$$

We choose as first approximation for our fundamental solution

$$
\begin{aligned}
& u_{0}(x, y, t ; \xi, \eta, \tau) \\
& \quad=[\phi(\xi, \eta, \tau)]^{-1}(\tau-t)^{-2 n} \exp \left[-\sum_{i, k} A_{i k}(x, y, t) R_{i k}(x, y, t ; \xi, \eta, \tau)\right] .
\end{aligned}
$$

$\phi(x, y, t)$ is defined by

$$
\begin{aligned}
& \phi(x, y, t)=\lim _{\lambda \rightarrow t_{+}} \int_{Q}(\lambda-t)^{-2 n} \\
& \cdot \exp \left[-\sum_{i, k} A_{i k}(x, y, t) R_{i k}(x, y, t ; \mu, \nu, \lambda)\right] d \mu d \nu .
\end{aligned}
$$

$Q$ is a small square centered in the point $x$. By a simple change of variable this limit can be shown to exist. By Lemma 1 and assumption (a) it follows that $\phi$ is a continuous function bounded away from zero and differentiable with respect to all its variables.

In the following we shall determine a function $f(\mu, \nu, \lambda ; \xi, \eta, \tau)$ such that the fundamental solution of (1.1) can be written as

$$
\begin{aligned}
u(x, y, t ; \xi, \eta, \tau)= & u_{0}(x, y, t ; \xi, \eta, \tau) \\
& +\int_{t}^{r} d \lambda \int_{R} u_{0}(x, y, t ; u, \nu, \lambda) f(\mu, \nu, \lambda ; \xi, \eta, \tau) d \mu d \nu
\end{aligned}
$$


For this purpose we need a set of three formulas collected in the following lemma.

Lemma 2. Let $f(\mu, \nu, \lambda)$ satisfy a Lipschitz condition of order $\gamma, 0<\gamma$, for any point $(\mu, \nu)$ in $R$ and $t \leqq \lambda<\tau$. For any $\epsilon>0$ let $f(\mu, \nu, \lambda)$ be bounded over any set for $\lambda \leqq \tau-\epsilon$, and absolutely integrable over $R$ for $t \leqq \lambda \leqq \tau$.

Let

$$
U(x, y, t)=\int_{t}^{\tau} d \lambda \int_{R} f(\mu, \nu, \lambda) v_{0}(x, y, t ; \mu, \nu, \lambda) d \mu d \nu
$$

with $t_{0} \leqq t<\tau \leqq t_{1}$ and $v_{0}(x, y, t ; \mu, \nu, \lambda)=u_{0}(x, y, t ; \mu, \nu, \lambda) \phi(\mu, \nu, \lambda)$. Then we have

$$
\begin{aligned}
\frac{\partial U}{\partial t}= & -f(x, y, t) \phi(x, y, t) \\
& +\int_{t}^{\tau} d \lambda \int_{R}[f(\mu, \nu, \lambda)-f(x, y, t)] \frac{\partial v_{0}}{\partial t} d \mu d \nu \\
& +f(x, y, t) \int_{\rightarrow t_{+}}^{\tau} d \lambda \int_{R} \frac{\partial v_{0}}{\partial t} d \mu d \nu \\
\frac{\partial U}{\partial y_{k}}= & \int_{t}^{\tau} d \lambda \int_{R}[f(\mu, \nu, \lambda)-f(x, y, t)] \frac{\partial v_{0}}{\partial y_{k}} \\
& +f(x, y, t) \int_{\rightarrow t_{+}}^{\tau} d \lambda \int_{R} \frac{\partial v_{0}}{\partial y_{k}} d \mu d \nu, \\
\frac{\partial^{2} U}{\partial x_{i} \partial x_{k}}= & \int_{t}^{\tau} d \lambda \int_{R}[f(\mu, \nu, \lambda)-f(x, y, t)] \frac{\partial^{2} v_{0}}{\partial x_{i} \partial x_{k}} d \mu d \nu \\
& +f(x, y, t) \int_{\rightarrow t_{+}}^{\tau} d \lambda \int_{R} \frac{\partial^{2} v_{0}}{\partial x_{i} \partial x_{k}} d \mu d \nu .
\end{aligned}
$$

Each of the last integrals means $\lim _{\epsilon \rightarrow 0} \int_{i+e}^{\tau}$.

Equation (3.11) is an extension of Theorem 1 of Dressel [4] and (3.13) of Theorem 2. Our fundamental solution differs from his by the normalization factor and the second exponential term.

Proof of (3.11). Let $Q$ be a $2 n$-dimensional square of side length $2 \eta$ and $R-Q$ the remainder of the region considered. We write for $\Delta t>0$

$$
\frac{\Delta_{t} U}{\Delta t}=-\frac{1}{\Delta t} \int_{t}^{t+\Delta t} d \lambda \int_{R} f v_{0} d \mu d \nu+\int_{t+\Delta t}^{\tau} d \lambda \int_{R} f \frac{\Delta_{t} v_{0}}{\Delta t} d \mu d \nu=I_{1}+I_{2} .
$$

To shorten the formulas we omit the variables on which depend $U, f, v_{0}$; they are to be found explicitly in (3.10). $\Delta_{t}$ means an increment where $t$ alone is 
varied.

The part of $I_{1}$ for which the space integral extends over $R-Q$ tends to zero with $\Delta t$. The remaining integral, with the space integration over $Q$, tends to $-f(x, y, t) \phi(x, y, t)$, because of the definition of $\phi$ and the continuity of $f$ in the point $(x, y, t) . I_{2}$ can be split up into three parts, the first an integration over $R-Q$ and from $t+\Delta t$ to $\tau-\epsilon$, the second integrated over $R$ and from $\tau-\dot{\epsilon}$ to $\tau$, and the third over $Q$ and from $t+\Delta t$ to $\tau-\epsilon$, where $\tau-\epsilon>t+\Delta t$ and $\epsilon>0$. In the first two integrals we can pass to the limit $\Delta t \rightarrow 0$ under the integral sign. For the third integral we get

$$
\begin{aligned}
J= & \int_{t+\Delta t}^{\tau-\epsilon} d \lambda \int_{Q}[f(\mu, \nu, \lambda)-f(x, y, t)] \frac{\Delta_{t} v_{0}}{\Delta t} d \mu d \nu \\
& +f(x, y, t) \int_{t+\Delta t}^{\tau-\epsilon} d \lambda \int_{Q} \frac{\Delta_{t} v_{0}}{\Delta t} d \mu d \nu=J_{1}+J_{2} .
\end{aligned}
$$

The Lipschitz condition on $f$ ensures the existence and convergence to zero with $\epsilon$ of

$$
\int_{t}^{t+\epsilon} d \lambda \int_{Q}[f(\mu, \nu, \lambda)-f(x, y, t)] \frac{\partial v_{0}}{\partial t} d \mu d \nu
$$

which implies that one can pass to the limit under the integral sign in $J_{1}$. We now show that $\lim _{\Delta_{t \rightarrow 0}} J_{2}$ exists. Consider

$$
J_{3}=f(x, y, t) \int_{t+\Delta t}^{t+\Delta t+\epsilon} d \lambda \int_{Q} \frac{\Delta_{t} v_{0}}{\Delta t} d \mu d \nu
$$

In the integral obtained by subtracting $J_{3}$ from $J_{2}$ one can pass to the limit under the integral sign. In $J_{3}$ we change $\lambda$ into $\lambda+\Delta t$ in $v_{0}(x, y, t+\Delta t ; \mu, \nu, \lambda)$ and obtain

$$
\begin{aligned}
J_{3}= & f(x, y, t)\left\{\int_{t}^{t+\Delta t} d \lambda / \Delta t \int_{Q}(\lambda-t)^{-2 n}\right. \\
& \cdot \exp \left[-\sum_{i, k} A_{i k}(x, y, t+\Delta t) R_{i k}(x, y, t ; \mu, \nu, \lambda)\right] d \mu d \nu+\int_{t+\Delta t}^{t+\Delta t+\epsilon} d \lambda / \Delta t \\
& \cdot \int_{Q} \frac{\exp \left[-\sum_{i, k} A_{i k}(x, y, t+\Delta t) R_{i k}\right]-\exp \left[-\sum_{i, k} A_{i k}(x, y, t) R_{i k}\right]}{(\lambda-t)^{2 n}} d \mu d \nu \\
& \left.-\int_{t+\epsilon}^{t+\Delta t+\epsilon} d \lambda / \Delta t \int_{Q}(\lambda-t)^{-2 n} \exp \left[-\sum_{i, k} A_{i k}(x, y, t+\Delta t) R_{i k}\right] d \mu d \nu\right\} .
\end{aligned}
$$

Passing to the limit $\Delta t$ tending to zero, we get 


$$
\begin{aligned}
\lim _{\Delta t \rightarrow 0} J_{3}= & f(x, y, t) \phi(x, y, t)+f(x, y, t) \int_{t}^{t+\epsilon} d \lambda \int_{Q}-\sum_{i, k} \frac{\partial A_{i k}}{\partial t} R_{i k} v_{0} d \mu d \nu \\
& -f(x, y, t) \int_{Q} v_{0}(x, y, t ; \mu, \nu, t+\epsilon) d \mu d \nu .
\end{aligned}
$$

The passage to the limit $\epsilon \rightarrow 0$ completes the proof of (3.11) for $\Delta t>0$. For $\Delta t<0$ we write

$$
\frac{\Delta_{t} U}{\Delta t}=\int_{t+\Delta t}^{t} d \lambda / \Delta t \int_{R} f v_{0}(x, y, t+\Delta t ; \mu, \nu, \lambda) d \mu d \nu-\int_{t}^{\tau} d \lambda \int_{R} f \frac{\Delta_{t} v_{0}}{\Delta t} d \mu d \nu
$$

and follow a proof completely analogous to the preceding one.

Proof of (3.12). Let $\epsilon>0$ and $t+\epsilon<\tau-\epsilon$. We form the ratio $\Delta_{y} U / \Delta y_{k}$ and split the integration from $t$ to $\tau$ into three parts, from $\tau-\epsilon$ to $\tau, t+\epsilon$ to $\tau-\epsilon$, and $t$ to $t+\epsilon$, thus obtaining three integrals of which the first two are regular. In these the limit can be taken under the integral sign. The third can be transformed into

$$
\int_{t}^{t+\epsilon} d \lambda \int_{R}[f(\mu, \nu, \lambda)-f(x, y, t)] \frac{\Delta_{y_{k} v_{0}}}{\Delta y_{k}} d \mu d \nu+f(x, y, t) \int_{t}^{t+\epsilon} d \lambda \int_{R} \frac{\Delta_{y_{k} v_{0}}}{\Delta y_{k}} d \mu d \nu .
$$

Because of the Lipschitz condition on $f(x, y, t)$, the passage to the limit can be effected under the first integral. Its contribution tends to zero with $\epsilon$. Introducing in the second the new variable $\bar{\nu}_{k}=\nu_{k}-\Delta y_{k}$ and calling $\bar{R}$ the transformed region, we obtain

$$
\begin{aligned}
& f(x, y, t) \int_{t}^{t+\epsilon} d \lambda \\
& \quad \int_{\bar{R}} \frac{\exp \left[-\sum_{i, e} A_{i e}\left(x, y_{k}+\Delta y_{k}, t\right) R_{i e}\right]-\exp \left[-\sum_{i, e} A_{i e}(x, y, t) R_{i, e}\right]}{\Delta y_{k}(\lambda-t)^{2 n}} d \mu d \nu .
\end{aligned}
$$

We let $\Delta y_{k} \rightarrow 0$ and obtain

$$
f(x, y, t) \int_{t}^{t+\epsilon} d \lambda \int_{R}-\sum_{i, e} \frac{\partial A_{i e}}{\partial y_{k}} R_{i e} v_{0} d \mu d \nu .
$$

Now we let $\epsilon \rightarrow 0$ and, combining the results of this paragraph, we obtain (3.12).

Proof of (3.13). It is easy to see that $\partial U / \partial x_{k}$ can be obtained by a differentiation under the integral sign. It is sufficient therefore to examine the derivative with respect to $x_{m}$ of

$$
T=\int_{t}^{t+\epsilon} d \lambda \int_{Q} f(\mu, \nu, \lambda) R_{e}(x, y, t ; \mu, \nu, \lambda) v_{0}(x, y, t ; \mu, \nu, \lambda) d \mu d \nu
$$


where we let

$$
\begin{aligned}
R_{e}= & -\sum_{i, k} \frac{\partial A_{i k}(x, y, t)}{\partial x_{e}} R_{i k}(x, y, t ; \mu, \nu, \lambda) \\
& +\sum_{i} A_{i e}\left[2 \frac{\mu_{i}-x_{i}}{4(\lambda-t)}-3 \frac{\nu_{i}-y_{i}-2^{-1}\left(\mu_{i}+x_{i}\right)(\lambda-t)}{(\lambda-t)^{2}}\right] .
\end{aligned}
$$

We increase $x_{m}$ by $\Delta x$ and keep all the other variables fixed; then we have

$$
\begin{aligned}
\frac{\Delta_{x} T}{\Delta x}=\frac{1}{\Delta x} \int_{t}^{t+\epsilon} d \lambda \int_{Q} f R_{e}\left(x_{m}+\Delta x, y, t ; \mu, \nu, \lambda\right)\left[v_{0}\left(x_{m}+\Delta x, y, t ; \mu, \nu, \lambda\right)\right. \\
\left.-\bar{v}_{0}\right] d \mu d \nu+\frac{1}{\Delta x} \int_{t}^{t+\epsilon} d \lambda \int_{Q} f\left[R_{e}\left(x_{m}+\Delta x, y, t ; \mu, \nu, \lambda\right) \bar{v}_{0}\right. \\
\left.-R_{e}(x, y, t ; \mu, \nu, \lambda) v_{0}(x, y, t ; \mu, \nu, \lambda)\right] d \mu d \nu
\end{aligned}
$$

where $\bar{v}_{0}$ denotes the function obtained from $v_{0}(x, y, t ; \mu, \nu, \lambda)$ by replacing the $x_{m}$ occurring in $A_{i k}(x, y, t)$ by $x_{m}$ and the $x_{m}$ elsewhere by $x_{m}+\Delta x$. To the first integral apply the mean value theorem and pass to the limit under the integral sign. It is easy to see that this integral tends to zero with $\epsilon$. In the other integral write for $f(\mu, \nu, \lambda)$ the sum $[f(\mu, \nu, \lambda)-f(x, y, t)]+f(x, y, t)$ and in this way obtain two integrals. Because of the Lipschitz condition on $f$, we can pass to the limit under the first integral. This integral then tends to zero with $\epsilon$. In the second integral $I_{2}$ we introduce the variables $\bar{\mu}_{m}=\mu_{m}-\Delta x$, $\bar{\nu}_{m}=\nu_{m}-2(\lambda-t) \Delta x$. Writing $Q^{\prime}$ for those integration limits in the integral which remain unchanged, we obtain.

$$
\begin{aligned}
I_{2}= & f(x, y, t) / \Delta x \int_{t}^{t+\epsilon} d \lambda \\
& \cdot \int_{Q^{\prime}}\left\{\int_{x_{m}-\eta-\Delta x}^{x_{m}+\eta-\Delta x} \int_{y_{m}-\eta-2 \Delta x(\lambda-t)}^{y_{m}+\eta-2 \Delta x(\lambda-t)}-\int_{x_{m}-\eta}^{x_{m}+\eta} \int_{y_{m}-\eta}^{y_{m}+\eta}\right\} R_{e} v_{0} d \mu d \nu \\
= & f(x, y, t) / \Delta x \int_{t}^{t+\epsilon} d \lambda \int_{Q^{\prime}}^{y_{m}}\left\{\int_{x_{m}-\eta-\Delta x}^{x_{m}+\eta-\Delta x}\left[\int_{y_{m}-\eta-2 \Delta x(\lambda-t)}^{y_{m}-\eta}-\int_{y_{m}+\eta-2 \Delta x(\lambda-t)}^{y_{m}+\eta}\right]\right. \\
& \left.+\int_{y_{m}-\eta}^{y_{m}+\eta}\left[\int_{x_{m}-\eta-\Delta x}^{x_{m}-\eta}-\int_{x_{m}+\eta-\Delta x}^{x_{m}+\eta}\right]\right\} R_{e} v_{0} d \mu d \nu .
\end{aligned}
$$

If $\Delta x$ is small enough, that is, satisfying $\Delta x<\min [n / 2, \eta / 4(\lambda-t)]$, the integrand is continuous and we can pass to the limit $\Delta x \rightarrow 0$ which gives us

$$
\lim _{\Delta x \rightarrow 0} I_{2}=f(x, y, t) \int_{t}^{t+\epsilon} d \lambda \int_{Q}\left[\frac{\partial R_{e}}{\partial \nu_{m}}+\frac{\partial R_{e}}{\partial \mu_{m}}\right] v_{0} d \mu d \nu .
$$


This gives the essential points in the proof of (3.13).

Lemma 3. The function $U$ defined by (3.10) satisfies the equation

$$
L(U)=-f(x, y, t) \phi(x, y, t)+\int_{t}^{\tau} d \lambda \int_{R} f(\mu, \nu, \lambda) L\left(v_{0}\right) d \mu d \nu,
$$

where $L$ is the operator defined in (1.1)

Proof. $L\left(v_{0}\right)$ can be written

$$
\begin{aligned}
(\lambda-t)^{2 n} L\left(v_{0}\right)= & \left\{\sum _ { i , k } R _ { i k } \left(-\sum_{e, m} \frac{\partial^{2} A_{i k}}{\partial x_{e} \partial x_{m}} a_{e m}+\sum_{e, m, r, s} \frac{\partial A_{i k}}{\partial x_{e}} \frac{\partial A_{r s}}{\partial x_{m}} a_{e m} R_{r s}\right.\right. \\
& \left.\left.-\sum_{e} \frac{\partial A_{i k}}{\partial x_{e}} a_{e}\right)+a\right\} \exp \left[-\sum_{i, k} A_{i k} R_{i k}\right] \\
& +(\lambda-t)^{-1 / 2}\left\{\sum _ { i } \left[\frac{\mu_{i}-x_{i}}{2(\lambda-t)^{1 / 2}}\right.\right. \\
& \left.+3 \frac{\nu_{i}-y_{i}-2^{-1}\left(\mu_{i}+x_{i}\right)(\lambda-t)}{(\lambda-t)^{3 / 2}}\right] \\
& \left.\cdot\left[2 \sum_{e, m} a_{e m} \frac{\partial A_{i m}}{\partial x_{e}}-4 \sum_{r, k, e, m} a_{e m} \frac{\partial A_{r k}}{\partial x_{e}} A_{i m} R_{r k}+\sum_{\bullet} a_{e} A_{i e}\right]\right\} \\
& \cdot \exp \left[-\sum_{i, k} A_{i k} R_{i k}\right] .
\end{aligned}
$$

The terms of higher order, that is, those containing $(\lambda-t)^{-2 n-1}$ and $(\lambda-t)^{-(2 n+3 / 2)}$, disappear because of the choice of $u_{0}$. This enables us to operate under the integral sign and derive (3.14) by Lemma 2.

We are now ready to construct $f(\mu, \nu, \lambda ; \xi, \eta, \tau)$ of formula (3.9) as the solution of the integral equation

$$
\begin{aligned}
& f(x, y, t ; \xi, \eta, \tau) \\
& \quad=L\left(u_{0}\right)+\int_{t}^{\tau} d \lambda \int_{R} L\left[u_{0}(x, y, t ; \mu, \nu, \lambda)\right] f(\mu, \nu, \lambda ; \xi, \eta, \tau) d \mu d \nu .
\end{aligned}
$$

In successive approximations we write:

$$
\begin{aligned}
f_{0}(x, y, t ; \xi, \eta, \tau) & =L\left(u_{0}\right), \\
f_{m}(x, y, t ; \xi, \eta, \tau) & =\int_{t}^{\tau} d \lambda \int_{R} L\left[u_{0}(x, y, t ; \xi, \eta, \tau)\right] f_{m-1}(\mu, \nu, \lambda ; \xi, \eta, \tau) d \mu d \nu \\
& (m \geqq 1),
\end{aligned}
$$

and put 


$$
f(x, y, t ; \xi, \eta, \tau)=\sum_{k=0}^{\infty} f_{k}(x, y, t ; \xi, \eta, \tau) .
$$

We prove first uniform and absolute convergence of the series in (3.17).

Let

$$
G_{i}(x, y, t ; \xi, \eta, \tau)=\left[\frac{\left(\xi_{i}-x_{i}\right)^{2}}{4(\tau-t)}+3 \frac{\left[\eta_{i}-y_{i}-2^{-1}\left(\xi_{i}+x_{i}\right)(\tau-t)\right]^{2}}{(\tau-t)^{3}}\right] .
$$

By (3a), Lemma 1, and (3.15) there are constants $d$ and $M$ such that

$$
\left|f_{0}\right| \leqq M(\tau-t)^{-2 n-1 / 2} \exp \left[-d^{2} \sum_{i} G_{i}(x, y, t ; \xi, \eta, \tau)\right]
$$

at fixed $\xi$, $\eta$, and $t<\tau$. In order to compute bounds on the terms $f_{k}$, we need an estimate on the integral

$$
\begin{aligned}
I= & \int_{t}^{\tau} d \lambda \int_{R}(\lambda-t)^{-2 n-1 / 2}(\tau-\lambda)^{-2 n-1 / 2} \\
& \cdot \exp \left\{-d^{2} \sum_{i}\left[G_{i}(x, y, t ; \mu, \nu, \lambda)+G_{i}(\mu, \nu, \lambda ; \xi, \eta, \tau)\right]\right\} d \mu d \nu .
\end{aligned}
$$

We change $d \mu_{i}=\mathrm{M}_{i}, d \nu_{i}=\mathrm{N}_{i}$, and in the same way $x_{i}, y_{i}, \xi_{i}, \eta_{i}$ into $d^{-1} X_{i}$, $d^{-1} Y_{i}, d^{-1} \Xi_{i}, d^{-1} \mathrm{H}_{i}$. The integral becomes

$$
\begin{aligned}
I= & d^{-2 n} \int_{t}^{\tau}(\lambda-t)^{-1 / 2}(\tau-\lambda)^{-1 / 2} d \lambda \int_{R}(\lambda-t)^{-2 n}(\tau-\lambda)^{-2 n} \\
& \cdot \exp \left\{-\sum_{i}\left[G_{i}(X, Y, t ; \mathrm{M}, \mathrm{N}, \lambda)+G_{i}(\mathrm{M}, \mathrm{N}, \lambda ; \Xi, \mathrm{H}, \tau)\right]\right\} d \mathrm{M} d \mathrm{~N} .
\end{aligned}
$$

$I$ is less than or equal to the integral obtained by replacing $R$ by the whole phase-space $S$. We also know that the function defined in (3.5) satisfies the corollary of Theorem 1 . Now the integral over $S$ is nothing but a product of integrals as in (2.4) and therefore we get

$$
\begin{aligned}
I \leqq & \left(3^{1 / 2} d\right)^{-2 n}(2 \pi)^{2 n} \int_{t}^{\tau}(\lambda-t)^{-1 / 2}(\tau-\lambda)^{-1 / 2}(\tau-t)^{-2 n} \\
& \cdot \exp \left[-\sum_{i} G_{i}(X, Y, t ; \Xi, \mathrm{H}, \tau)\right] d \lambda, \\
I \leqq & \left(d 3^{1 / 2}\right)^{-2 n}(2 \pi)^{2 n} \pi(\tau-t)^{-2 n} \exp \left[-d^{2} \sum_{i} G_{i}(x, y, t ; \xi, \eta, \tau)\right] .
\end{aligned}
$$

Therefore

$$
\left|f_{1}\right| \leqq \pi\left(d 3^{1 / 2}\right)^{-2 n}(2 \pi)^{2 n} M^{2}(\tau-t)^{-2 n} \exp \left[-d^{2} \sum_{i} G_{i}(x, y, t ; \xi, \eta, \tau)\right] .
$$


Estimates on the remaining terms of the series are obtained by induction. We get

$$
\left|f_{k}\right| \leqq \pi^{(k-1) / 2}\left(d 3^{1 / 2}\right)^{-2 n k}(2 \pi)^{2 n k} M^{k+1}(\tau-t)^{1 / 2(k-1)-2 n} \frac{\exp \left[-d^{2} \sum_{i} G_{i}\right]}{\Gamma(k / 2+1 / 2)}
$$
and therefore

$$
|f| \leqq \text { const }(\tau-t)^{-(2 n+1 / 2)} \exp \left[-d^{2} \sum_{i} G_{i}\right] .
$$

For $t \leqq \tau-\epsilon$ the series (3.17) is uniformly and absolutely convergent. Therefore (3.17) defines a continuous function for $t \leqq \tau-\epsilon$ and by (3.19) this function is absolutely integrable over $R$ for $t<\tau$. We still have to prove that the function $f$ satisfies a Lipschitz condition of order $\gamma, 0<\gamma$. Because of (3a) and (3.15), $L\left(u_{0}\right)$ satisfies a Lipschitz condition. It is therefore sufficient to prove that

$$
f^{*}(x, y, t)=\int_{t}^{\tau} d \lambda \int_{R} L\left[u_{0}(x, y, t ; \mu, \nu, \lambda)\right] f(\mu, \nu, \lambda ; \xi, \eta, \tau) d \mu d \nu
$$

satisfies a Lipschitz condition.

We keep $x_{k}, k \neq i$, fixed and write for $x_{i}^{(1)}<x_{i}^{(2)}$, both in $R$,

$$
f^{*}\left(x_{i}^{(1)}, y, t\right)-f^{*}\left(x_{i}^{(2)}, y, t\right)=\int_{t}^{\tau} d \lambda \int_{R} \Delta L\left(u_{0}\right) f d \mu d \nu,
$$

where we introduce the notation

$$
\Delta L\left(u_{0}\right)=L\left[u_{0}\left(x_{i}^{(1)}, y, t ; \mu, \nu, \lambda\right)\right]-L\left[u_{0}\left(x_{i}^{(2)}, y, t ; \mu, \nu, \lambda\right)\right] .
$$

Outside the region $E$

$$
E\left\{\begin{array}{lr}
x_{i}^{(1)} \leqq \mu \leqq x_{i}^{(2)}, & \\
t \leqq \lambda \leqq t+a & (a>0, t+a<\tau), \\
y-b \leqq \nu \leqq y+b & (b>0), \\
x_{k}-b \leqq \mu_{k} \leqq x_{k}+b & (k \neq i),
\end{array}\right.
$$

$L\left(u_{0}\right)$ satisfies a Lipschitz condition and $f(\mu, \nu, \lambda ; \xi, \eta, \tau)$ is absolutely integrable. Therefore it is sufficient to show that

$$
N \int_{t}^{t+a} d \lambda \int_{E}\left|\Delta L\left(u_{0}\right)\right| d \mu d \nu
$$

satisfies a Lipschitz condition, $N$ is the bound on $f(\mu, \nu, \lambda ; \xi, \eta, \tau)$ in $R$. 
According to (3.15)

$$
\begin{aligned}
\left|\Delta L\left(u_{0}\right)\right| \leqq & \text { const. }(\lambda-t)^{-2 n-1 / 2}\left\{\exp \left[-d^{2} \sum_{k} G_{k}\left(x_{i}^{(1)}, y, t ; \mu, \nu, \lambda\right)\right]\right. \\
& \left.-\exp \left[-d^{2} \sum_{k} G_{k}\left(x_{i}^{(2)}, y, t ; \mu, \nu, \lambda\right)\right]\right\} .
\end{aligned}
$$

We split $E$ into two parts according to

$$
x_{i}^{(1)} \leqq \mu \leqq\left(x_{i}^{(1)}+x_{i}^{(2)}\right) / 2, \quad\left(x_{i}^{(1)}+x_{i}^{(2)}\right) / 2 \leqq \mu \leqq x_{i}^{(2)} .
$$

We divide the integral inside by $\left(\mu-x_{i}^{(1)}\right)^{\gamma}$ and $\left(x_{i}^{(2)}-\mu\right)^{\gamma}, 0<\gamma<1$, respectively and multiply outside both integrals by $\left|x_{i}^{(1)}-x_{i}^{(2)}\right|^{\gamma}$. We obtain bounded integrals and have

$$
\left|\int_{t}^{t+a} d \lambda \int_{E} f \Delta L\left(u_{0}\right) d \mu d \nu\right| \leqq \text { const. }\left|\stackrel{(1)}{x_{i}}-\stackrel{(2)}{x_{i}}\right|^{\gamma} .
$$

For $y_{i}^{(1)}-y_{i}^{(2)}$ the proof is analogous.

For $t<t^{(1)}<t^{(2)}<\tau$ let us consider

$$
\begin{aligned}
u\left(x, y, t^{(1)}\right)-u\left(x, y, t^{(2)}\right)= & \int_{t^{(1)}}^{\tau} d \lambda \int_{R} f L\left[u_{0}\left(x, y, t^{(1)} ; \mu, \nu, \lambda\right)\right] d \mu d \nu \\
& -\int_{t^{(2)}}^{\tau} d \lambda \int_{R} f L\left[u_{0}\left(x, y, t^{(2)} ; \mu, \nu, \lambda\right)\right] d \mu d \nu .
\end{aligned}
$$

We write

$$
\begin{aligned}
u\left(x, y, t^{(1)}\right)-u\left(x, y, t^{(2)}\right)= & \int_{t^{(1)}}^{m} d \lambda \int_{R} f L\left[u_{0}\left(x, y, t^{(1)} ; \mu, \nu, \lambda\right)\right] d \mu d \nu \\
& +\int_{t^{(2)}}^{m} d \lambda \int_{R} f L\left[u_{0}\left(x, y, t^{(2)} ; \mu, \nu, \lambda\right)\right] d \mu d \nu \\
& +\int_{m}^{\tau} d \lambda \int_{R} f\left\{\left[u_{0}\left(x, y, t^{(1)} ; \mu, \nu, \lambda\right)\right]\right. \\
& \left.-L\left[u_{0}\left(x, y, t^{(2)} ; \mu, \nu, \lambda\right)\right]\right\} d \mu \nu
\end{aligned}
$$

where $m=$ minimum of $\left(3 t^{(2)}-t^{(1)}\right) / 2$ and $\tau$.

The last integral satisfies a Lipschitz condition, because $L\left(u_{0}\right)$ does and $f$ is absolutely integrable.

The first two integrals are bounded and give

$$
\mid u\left(x, y, t^{(1)}\right)-u\left(x, y, t^{(Q)} \mid \leqq \text { const. }\left|t^{(2)}-t^{(1)}\right|^{1 / 2} .\right.
$$

We have therefore proved that $f$ satisfies a Lipschitz condition. 
We now write (3.9) and prove that $u$ thereby defined satisfies properties I-III of $\$ 2$ and this will complete the proof of Theorem 2.

(I) The result of the last paragraph, formula (3.9), and Lemma 3 enable us to write

$$
L(u)=L\left(u_{0}\right)-f(x, y, t ; \xi, \eta, \tau)+\int_{t}^{\tau} d \lambda \int_{R} L\left(u_{0}\right) f(\mu, \nu, \lambda ; \xi, \eta, \tau) d \mu d \nu,
$$

which gives, by (3.16), $L(u)=0$. We notice that for $t \leqq \tau-\epsilon, u_{0}$ and its derivatives are continuous. So is $\partial u / \partial x_{k}$, which can be verified by direct differentiation under the integral sign. We need to show only that $\partial^{2} u / \partial x_{k} \partial x_{r}$ and $\partial u / \partial y_{r}$ are continuous. This will entail by (1.1) continuity of $\partial u / \partial t$. It is sufficient to examine for continuity formulas (3.12) and (3.13). We can write (3.12)

$$
\begin{aligned}
\frac{\partial U}{\partial y_{k}}= & \lim _{\epsilon \rightarrow 0} \int_{t+\epsilon}^{\tau-\epsilon} d \lambda \int_{R} \frac{\partial v_{0}}{\partial y_{k}}[f(\mu, \nu, \lambda)-f(x, y, t)] d \mu d \nu \\
& +\lim _{\epsilon \rightarrow 0} f(x, y, t) \int_{t+\epsilon}^{\tau-\epsilon} d \lambda \int_{R} \frac{\partial v_{0}}{\partial y_{k}} d \mu d \nu .
\end{aligned}
$$

This limit is uniform in $(x, y, t)$ for $t \leqq \tau-\epsilon$. Let $(x, y, t)$ tend to $(X, Y, T)$, $(X, Y)$ in $R$ and $T \leqq \tau$. We can interchange the limits $\epsilon \rightarrow 0$ and $\lim (x, y, t)$ $=(X, Y, T)$ and this latter can be taken under the integral sign. This proves continuity of $\partial U / \partial y_{k}$. The proof is the same for $\partial^{2} U / \partial x_{i} \partial x_{k}$. Therefore property $I$ is satisfied by $u$.

(II) We have by (3.9) and (3.19)

$$
\begin{aligned}
\left|u-u_{0}\right| & \leqq \text { const. }\left[\phi_{\min }\right]^{-1} \int_{t}^{\tau}(\lambda-t)^{-2 n}(\tau-\lambda)^{-2 n-1 / 2} d \lambda \\
& \cdot \int_{R} \exp \left[-d^{2} \sum_{i} G_{i}(x, y, t ; \mu, \nu, \lambda)-d^{2} \sum_{i} G_{i}(\mu, \nu, \lambda ; \xi, \eta, \tau)\right] d \mu d \nu ;
\end{aligned}
$$

we get

$$
\left|u-u_{0}\right| \leqq \frac{\text { const. }}{\phi_{\min }} 2^{-1}(\tau-t)^{1 / 2} \exp \left[-d^{2} \sum_{i} G_{i}(x, y, t ; \xi, \eta, \tau)\right] .
$$

Therefore for any continuous and bounded function $f(x, y)$ we have

$$
\begin{aligned}
\lim _{t \rightarrow \tau-} \int_{D} u(x, y, t ; \xi, \eta, \tau) f(x, y) d x d y & \\
= & \lim _{t \rightarrow \tau-} \int_{D} u_{0}(x, y, t ; \xi, \eta, \tau) f(x, y) d x d y
\end{aligned}
$$


where $D$ is finite or infinite. The properties of $u_{0}$ immediately yield II.

(III) According to (3.19) we see that $u(x, y, t ; \xi, \eta, \tau)$ and $x_{i} u(x, y, t ; \xi, \eta, \tau)$ are absolutely integrable. Differentiation of (3.9) shows that $\partial u / \partial x_{k}$ are bounded. Hence the results of $\$ 2$ apply to our fundamental solution.

To illustrate the use of the fundamental solution we consider an initialvalue problem. If $R$ is the whole of phase space, the following simple problem can be solved: given a continuous bounded function $\psi(x, y)$ there is for $t>0$ a unique solution of equation (2.2) satisfying

$$
\lim _{t \rightarrow 0} u(x, y, t)=\psi(x, y),
$$

provided that both $u$ and $\partial u / \partial x_{k}$ are bounded and we have

$$
u(x, y, t)=\int_{-\infty}^{+\infty} \psi(\xi, \eta) u(\xi, \eta, \tau ; x, y, t) d \xi d \eta
$$

(3.22) is easily obtained by use of Green's formula. Uniqueness is consequence of the fact that for $\psi \equiv 0, u \equiv 0$. It is obvious that the restrictions on $u$ and $\partial u / \partial x_{k}$ can be relaxed, because of the fact that $u(x, y, t ; \xi, \eta, \tau)$ decreases exponentially as well as $\partial u / \partial x_{k}$ for large values of the coordinates.

\section{BIBLIOGRAPHY}

1. A. Kolmogoroff, Zufaellige Bewegungen, Ann. of Math. vol. 35 (1934).

2. S. Chandrasekhar, Stochastic processes in physics and astronomy, Reviews of Modern Physics vol. 15 I (1943).

3. W. Feller, Zur Theorie der stochastischen Prozesse, Math. Ann. vol. 113 (1936).

4. F. G. Dressel, The fundamental solution of the parabolic equation, Duke Math. J. vol. 7 (1940).

5. - - The fundamental solution of the parabolic equation, Duke Math. J. vol. 13 (1946).

6. M. Gevrey, Sur la nature analytique des solutions des équations aux dérivées partielles, Ann. Ecole Norm. vol. 35 (1918). 9 (1913).

7. ——Equations aux dérivées partielles du type parabolique, J. Math. Pures Appl. vol.

California Institute of Technology 